\title{
Roles of the Main Olfactory and Vomeronasal Systems in the Response of the Female Hamster to Young
}

\author{
David M. MARques ${ }^{1}$ \\ Department of Psychology, University of Michigan, Ann Arbor, Michigan 48109
}

\begin{abstract}
A series of experiments was undertaken to investigate the roles of the main olfactory and vomeronasal systems in the response of the female golden hamster (Mesocricetus auratus) to pups. Upon first encountering hamster pups, virgin females are either maternal or cannibalistic. Radical bulbectomy, which disrupts both the main and accessory bulbs, reduced maternal behavior, pup killing, and nest building. Many animals neither killed nor carried pups following radical bulbectomy, and some previously maternal animals attacked pups after bulbectomy. The effects of bulbectomies were not reproduced by peripheral deafferentations (sensory loss only) of the main olfactory and vomeronasal systems. After vomeronasal nerve cuts (VNNC) most killers became carriers and built better nests than before deafferentation. Zinc sulfate treatment (main olfactory system sensory loss) alone had little effect, but when combined with transection of the vomeronasal nerves, it converted to carriers those killers that had continued to kill after VNNC. Because none of these procedures affected cricket killing, the treatments that reduced pup killing appear to be stimulus rather than response related. The behavior pattern displayed during cricket killing was similar to that observed during pup killing.
\end{abstract}

Odors play an important role in controlling the female rodent's responses to pups. One way to investigate the role of olfaction in maternal behavior is to eliminate all olfactory stimulation by removing the olfactory bulbs. This procedure has produced complex effects on maternal behavior in rats.

${ }^{1}$ This research was supported in part by Research Grant 2 R01 MH20811-03 from the National Institute of Mental Health and NSF Research Grant BMS75-08595. I am indebted to Dr. Elliot S. Valenstein for guidance in all phases of the project, as well as to Drs. Sarah Winans, Neil Rowland, Brad Powers, and Chuck Malsbury for valuable criticisms on earlier drafts of the manuscript, and to Bonnie Marques for histological, secretarial, and graphical assistance. This research was conducted as partial fulfillment of the requirements of a doctoral degree in Psychology granted by the University of Michigan, and was supported in part by a dissertation grant to the author. Results have previously been reported at the meeting of the Society for Neuroscience, November 1976, and published in Neuroscience Abstracts, 2, 158 (1976). Send reprint requests to: The Worcester Foundation for Experimental Biology, Shrewsbury, Mass. 01545. 
Recent neuroanatomical findings have given rise to the concept of a dual olfactory system (Raisman, 1972). In rodents, the chemosensory receptors of the vomeronasal organ and those of the main olfactory mucosa have separate central connections (Davis, Macrides, Youngs, Schneider, \& Rosene, 1978 for hamster; Scalia \& Winans, 1976, review). This raises the possibility that the functions of the two systems may be different. Powers and Winans (1975) have demonstrated that cutting the vomeronasal nerves reduces sexual performance in the male hamster.

Olfactory bulbectomy interrupts both the vomeronasal and main olfactory sensory systems, and may have "nonsensory" effects as well (Cain, 1974). Bulbectomy produces complex changes in rodent maternal behaviors (Benuck \& Rowe, 1975; Fleming \& Rosenblatt, 1974a; Leonard, 1972; Murphy, 1972), but how these two systems contribute to the changes is presently unknown. Thus it is important to examine the effects of relatively selective disruptions of either the main olfactory or vomeronasal systems.

Many investigators have found less severe deficits in maternal behavior after zinc sulfate-induced anosmia than after bulb removal. Fleming and Rosenblatt (1974b) showed that, unlike bulbectomy, destruction of main olfactory receptors with zinc sulfate did not induce virgin rats to cannibalize pups, but instead reduced the latency for induction of pup retrieval. Benuck and Rowe (1975) observed no cannibalism in zinc sulfatetreated lactating rats, but did find slight deficits in retrieving. Vandenbergh (1973) reported that zinc sulfate-treated lactating mice show neglect, cannibalism, and deficits in nest building which are not as severe as those produced by bulbectomy. Experience with previous parturitions can overcome deficits in mice after zinc sulfate treatment (Seegal \& Denenberg, 1974) but not after bulbectomy (Gandelman, Zarrow, Denenberg, \& Myers, 1971).

The differences between bulbectomy and peripheral sensory loss could be due to the destruction of the vomeronasal system which accompanies bulbectomy but not (in some cases at least, i.e., Powers \& Winans, 1975; Winans \& Powers, 1977) zinc sulfate treatments. Only two recent papers (Powers \& Winans, 1975; Johns, Feder, Komisaruk, \& Meyer, 1978) have convincingly separated the behavior effects of damage to the main olfactory and vomeronasal systems in mammals. Relevant to this paper, Powers and Winans showed that the complete elimination of mating behavior in the male hamster seen after bulbectomy is reproduced by combined loss of both vomeronasal functioning and olfactory cues received via the olfactory mucosa.

Virgin hamsters display either pup killing or carrying immediately upon encountering young (Marques \& Valenstein, 1976; Rowell, 1961) and the dichotomy between "killers" and "carriers" is stable (Marques, 1976a; Marques \& Valenstein, 1976). In order to classify an animal in one of these two categories, all animals in the present study were pretested 
before surgery. Therefore, they are experienced killers and carriers. Conclusions relate to the effects of treatments on the "maintenance" of the response patterns in virgin females.

In this experiment, both killers and carriers were subjected to either olfactory bulbectomy or a peripheral sensory deafferentation. Animals in the latter groups received either vomeronasal deafferentation or olfactory deafferentation or both treatments. The combined treatment was intended to cause loss of all the main olfactory and vomeronasal sensory information which bulbectomy eliminates. A second experiment tested whether the reductions in pup killing observed after some treatments were due to a general response or motor deficit or due to a somewhat more selective change in response to pups.

\section{EXPERIMENT 1: OLFACTORY SYSTEMS AND RESPONSES TO PUPS}

\section{Method}

Subjects. One-hundred and six adult female golden hamsters (Mesocricetus auratus) (90-100 g), obtained from Engle (Farmersburg, Ind. 47850 ), were housed singly in stainless-steel cages $20 \times 25 \times 18 \mathrm{~cm}$ with wire-mesh bottoms. They were given food and water ad libitum but no nesting material and were kept on a 14-hr L: 10-hr D reversed light cycle (off at 9 AM). Experimental animals were housed in the same room with males but in a separate room from pregnant and lactating females. In their home cages, animals could hear, smell, and possibly see other females.

Apparatus. Subjects were tested in clear acrylic boxes, $45.7 \times 30.5 \times 30.5$ $\mathrm{cm}$ high. Behaviors were recorded on counters, running time meters, and an event recorder.

Procedure. Animals were given one preoperative test to determine their response to pups. Those which did not show the same behavior to both pups were not used in these experiments (7.2\%). Testing after treatment for the remaining 98 followed the same procedures as before treatment.

Treatment groups and testing schedule. Pretested hamsters were randomly assigned to one of five treatment groups. Animals receiving bilateral olfactory bulbectomy (14 carriers, 11 killers, preoperatively) and vomeronasal nerve cuts (VNNC; 10 carriers, 12 killers) were operated on within 1 week of preoperative tests and given postoperative tests $2-3$ weeks later. Two weeks after these tests, the VNNC animals were given zinc sulfate treatment and retested 2 or 3 days later. For this test, they are referred to as V-test-ZS animals. The third group received zinc sulfate treatment (ZS; 9 carriers, 7 killers) 2-3 weeks after pretests, with postoperative testing 2 or 3 days later, when anosmia was maximal for the ZS procedure used here (Powers \& Winans, 1973). A fourth group (V+ZStest; 9 carriers, 6 killers) was given vomeronasal nerve cuts 1 week after 
pretests and ZS treatment 1 week later. They were given their first postoperative test 2 or 3 days after this second treatment. All animals that had been treated with zinc sulfate were retested with pups 11 to 12 days later, when the anosmic effects of the treatment should have substantially worn off (see Powers \& Winans, 1973). This was the second postoperative test for animals in the $\mathrm{ZS}$ and $\mathrm{V}+\mathrm{ZS}$-test groups, and the third postoperative test for the V-test-ZS group. Five control animals were also given a second postoperative test at this time.

The control group comprised animals from all the different control procedures which had been combined because there were no differences among them. These controls included surgical controls for bulbectomy ( 5 carriers, 3 killers), vomeronasal deafferentation ( 3 carriers, 5 killers), and saline controls for zinc sulfate treatment ( 3 carriers, 2 killers).

Eleven of the twenty-five bulbectomized hamsters ( 5 postoperative killers, 1 postoperative carrier, 5 postoperative nonresponders) were tested a third time (the second postoperative test) 2-3 weeks after their first postoperative test, in order to determine if their altered response was consistent across repeated testing. Five controls were also tested a third time. Finally, the bulbectomized animals were placed with males for mating. Of the 25,18 ( 8 postoperative killers, 2 postoperative carriers, 8 postoperative nonresponders) delivered pups, 3 were mated but died during the last day of pregnancy, and 4 did not successfully mate in 10 consecutive days with males.

On Days 1 ( 24 hr after birth), 2, and 3 postpartum, surviving pups were scattered and mothers were observed for $5 \mathrm{~min}$. Pups still scattered after the tests were returned to the nests.

Operative procedures. For radical bulbectomies, animals were anesthetized with sodium pentobarbital $(75 \mathrm{mg} / \mathrm{kg}$, ip) and a flap of bone was lifted out above the olfactory bulbs. Main and accessory bulbs were removed bilaterally with aspiration, and an attempt was made to take out most of the olfactory peduncles. The empty cavity was then filled with hemostatic gauze. Controls received the same surgical treatment except aspiration of the bulbs.

To produce vomeronasal deafferentation (VNNC), the vomeronasal nerves were cut where they pass along the dorsomedial surface of the main olfactory bulbs. After animals were anesthetized and a bone flap was removed as for bulbectomy, an opened pair of small ophthalmic scissors (RS $638 \mathrm{McClure}$ ultramicro scissors) was lowered into the bulbs down to the cribriform plate. The scissors were removed after this for control treatment. To cut the nerves, the scissors were closed in a plane perpendicular to the nerves and pulled out. This resulted in extensive bleeding (stopped by pressure) from the bulb and the midline venus sinus. When the bleeding ceased, a piece of hemostatic gauze was placed on top of the bulbs and the skin sutured. 
The procedure used to destroy the olfactory mucosa with zinc sulfate has been described in detail elsewhere (Powers \& Winans, 1973). Briefly, ether-anesthetized subjects were placed on their backs, head down on an inclined plane. With the tongue reflected, a long hooked piece of polyethylene tubing attached to a hypodermic syringe was inserted through the mouth, and hooked anteriorly into the nasopharyngeal meatus. With continuous, light aspiration at the nares to prevent the solution from draining into the lungs, $0.5 \mathrm{ml}$ of $0.17 \mathrm{M}$ zinc sulfate solution in $0.5 \%$ saline was slowly pushed into the nasal cavities. This procedure destroys most of the main olfactory epithelium but leaves the vomeronasal epithlium histologically intact (Powers \& Winans, 1973; Winans $\&$ Powers, 1977). Controls received $0.5 \%$ saline delivered in exactly the same manner.

Testing with pups. Testing was done in dim red illumination during $\mathrm{hr}$ 2-6 of darkness. Animals were not tested on the day of behavioral estrus. Food and water from the animal's home cages and 10 strips of paper were placed with the subjects in the testing arenas. Up to seven hamsters were in the testing room together, visually isolated from each other.

After $1 \mathrm{hr}$ of adaptation and before the pups were introduced, animals were given nest quality (NQ) ratings of 0-2. A "2" required that the nesting paper be piled up in one corner and matted down, and a " 0 ", indicated no recognizable nest. Two pups (2-5 days old, obtained from donor mothers) were then introduced and the adult was observed for 10 min for the frequency, and in some cases duration, of 17 behavior patterns. Those with importance for this study include: lick, pick up, carry, bite or eat pup, drop pup in nest, carry or pull paper, carry food, and scrabble. Scrabbling is a typical hamster behavior that appears to be an escape behavior, as the animal stands on its hind legs and jumps up against the walls of the cage.

Maternal animals were also rated for carrying efficiency. The carrying efficiency quotient was obtained by dividing the number of times pups were placed in the nest by the number of times pups were picked up and placed anywhere.

After the testing period, the adult was returned to its home cage and surviving pups were returned to their mothers. These pups were not used again until 22-24 hr later. Reuse of pups on successive days was not a problem as there had been no change in proportions of carriers and killers across testing days in previous studies (Marques \& Valenstein, 1976; Marques, 1976a) and none was observed this time. Testing boxes were washed with detergent and dried before reuse.

Tests for olfaction. All animals which were bulbectomized or treated with zinc sulfate were deprived of food immediately after their tests with pups and observed $24 \mathrm{hr}$ later in one of the two different tests for assessing olfaction. Ten days later, all animals that had had ZS treatment were again 
tested for olfaction (to determine recovery from ZS treatment) and retested with pups. Tests were conducted in a dimly lit (one $25-\mathrm{W}$ red bulb) room during the fifth to sixth hour of the animals' dark cycle.

Food finding test. This was conducted in a small $(24 \times 30 \times 21 \mathrm{~cm}$ high $)$ plastic box, the floor of which was covered with $2-3 \mathrm{~cm}$ of sawdust. In the middle of one side, $3-4 \mathrm{~cm}$ from the wall, a single food pellet was buried. The measure of olfaction was the latency ( $300 \mathrm{sec}$ max) for the animal to pick up the pellet in its mouth or forepaws. All 16 animals in the ZS group and the 5 saline controls were tested in this procedure.

Food preference test. Other animals (bulbectomized, V+ZS-test, $\mathrm{V}$-test-ZS, and the remaining 16 controls) were tested in a more robust food preference test. The acrylic testing chamber $(23 \times 19 \times 27 \mathrm{~cm}$ high) had two holes, $1-\mathrm{cm}$ diam., drilled into the bottom, $7.5 \mathrm{~cm}$ from the two ends and $8 \mathrm{~cm}$ from each other. Under each hole was placed a $7.5-\mathrm{cm}$ deep glass jar, one containing two pieces of wood, the other containing two to three food pellets. Behavioral measurements included the latency and total duration (out of $200 \mathrm{sec}$ ) of sniffing or biting at each hole.

Histological analysis. After animals were perfused with $10 \%$ Formalin in $0.8 \%$ saline, skulls were decalcified in $25 \%$ formic acid for 3 days, then put into 5\% sodium sulfate for 1 day to neutralize the acid. Following a 24-hr wash in tap water, whole decalcified skulls were embedded in a $40^{\circ} \mathrm{C}$ mixture of two-thirds egg yolk and one-third $20 \%$ liquid gelatin. The matrix was then cooled and placed in $10 \%$ formalin for 1 week followed by 1 week of $30 \%$ sucrose in $10 \%$ Formalin. Some brains were sectioned horizontally and some coronally. All sections were $40 \mu \mathrm{m}$ thick and stained with cresyllecht violet.

Statistical analysis. Statistical analyses were based on two-tailed significance levels comparing the distributions of postoperative responses of each treatment group with the pooled control group, unless otherwise noted. Error estimates for means are SEM.

\section{Results}

\section{Histology}

The main and accessory bulbs were completely removed in all 16 radically bulbectomized animals examined histologically. The other bulbectomies were confirmed by dissection. Figure 1 shows coronal sections through the posterior part of the main olfactory bulb (a) and the olfactory peduncle (b-d). Most of the radically bulbectomized animals had very little normal olfactory tissue remaining rostral to the coronal plane of (c). The amount of damage to the peduncle in (c) and (d) of the lesion shown on the left is representative of the radically bulbectomized animals. The anterior tip of the olfactory tubercle was slightly damaged bilaterally in 


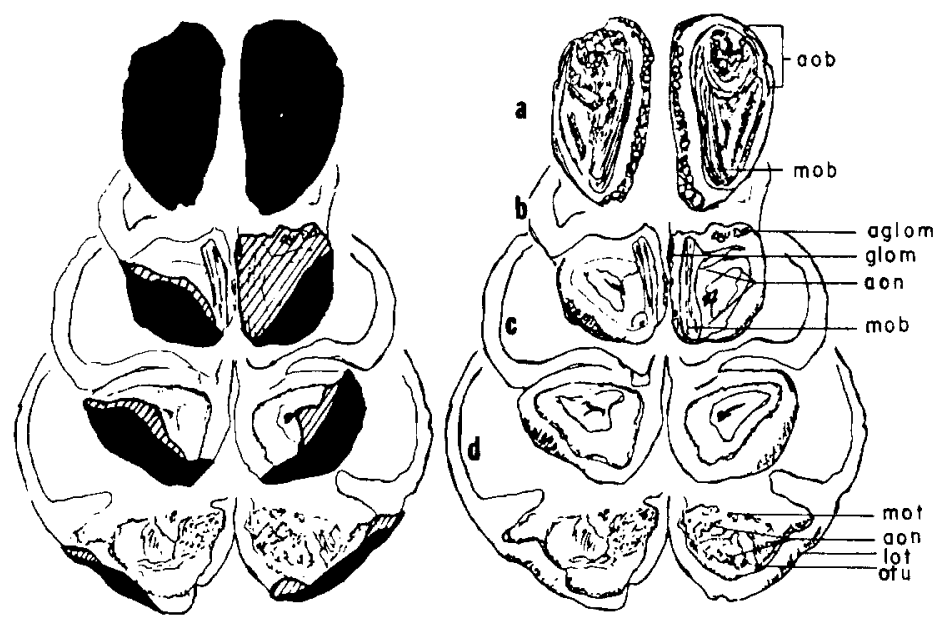

FIG. 1. Drawings show a representative lesion from the radical bulbectomy group, compared to normal tissue. Damage to the posterior main olfactory bulbs (a) and olfactory peduncle $(b-d)$ is indicated on coronal sections. The lesion shown is representative of most animals in the group. Black areas represent missing parts and hatched areas represent gliosis and absence of normal intracellular organization. Sections are $40 \mu \mathrm{m}$ thick, 12 sections apart, with section (a) most rostral and section (d) most caudal. Abbreviations: aob, accessory olfactory bulb; mob, main olfactory bulb; aon, anterior olfactory nucleus; (a)glom, glomeruli of the (accessory) main olfactory bulb; lot, lateral olfactory tract; mot, main olfactory tract; otu, olfactory tubercle.

three animals. There was no consistent correlation between the extent of tissue damage and postoperative behavior toward pups among bulbectomized animals.

All of the 23 animals in the VNNC or V+ZS groups which were evaluated histologically sustained complete vomeronasal nerve cuts reflected in the absence of any glomeruli in the accessory olfactory bulbs (Winans \& Powers, 1977). The range of additional damage done to the main olfactory bulbs is represented in Fig. 2 and varied around an estimated 5 to $15 \%$ of the main bulb tissue. The drawings are from coronal sections through the point of maximum damage in two animals. Most of the animals had intermediate amounts of destruction. The damage to main bulbs in these VNNC animals was restricted to the anterior one-third of the main bulbs, and had an average anterior-posterior length of 1.2-1.4 $\mathrm{mm}$.

\section{Behavior with Pups}

Controls. All 10 killer controls continued to kill after surgery and 9 of 11 carrier controls carried both pups presented in post-tests. The other 2 killed one pup and carried the other (Table 1).

Carriers improved from preoperative to postoperative tests. Decreases 


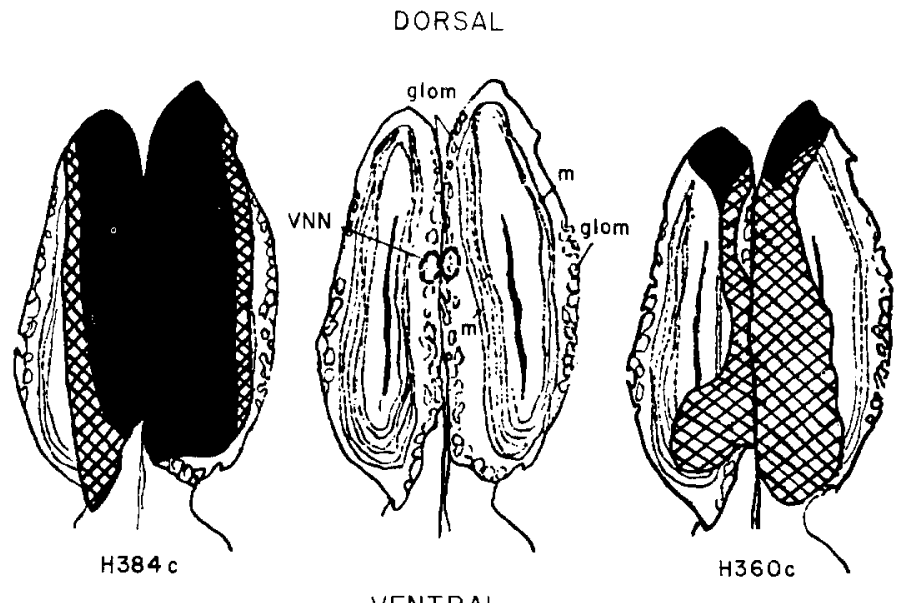

FIG. 2. Drawings of coronal sections illustrate the range of damage to the main olfactory bulbs following vomeronasal nerve cuts. One of the largest lesions is on the left, and a smaller one on the right, with a normal section in the middle. Most animals had intermediate amounts of damage. In all cases damage was restricted to the anterior one-third of the main bulbs. Abbreviations: glom, glomeruli; $\mathrm{m}$, mitral cell layer; VNN, approximate location of the vomeronasal nerves.

TABLE 1

Post-test Response to Pups of Pretest Carriers and Killers after Olfactory and Vomeronasal Disruptions ${ }^{a}$

\begin{tabular}{|c|c|c|c|c|c|}
\hline \multirow[b]{2}{*}{ Treatment } & \multirow[b]{2}{*}{$n$} & \multicolumn{4}{|c|}{ Post-test response $(\%)$} \\
\hline & & Kill & Carry & Neither & Both $^{b}$ \\
\hline \multicolumn{6}{|c|}{ Pretest carriers } \\
\hline Bulbectomy $\mathrm{y}^{c}$ & 14 & 28.6 & 7.1 & 64.3 & 0.0 \\
\hline ZS & 9 & 0.0 & 88.9 & 0.0 & 11.1 \\
\hline VNNC & 10 & 0.0 & 100.0 & 0.0 & 0.0 \\
\hline V-Test-ZS & 10 & 0.0 & 100.0 & 0.0 & 0.0 \\
\hline$V+Z S-T e s t$ & 8 & 0.0 & 87.5 & 0.0 & 12.5 \\
\hline Controls & 11 & 0.0 & 81.8 & 0.0 & 18.2 \\
\hline \multicolumn{6}{|c|}{ Pretest killers } \\
\hline Bulbectomy ${ }^{c}$ & 11 & 54.5 & 18.2 & 27.3 & 0.0 \\
\hline $\mathrm{ZS}$ & 7 & 71.4 & 28.6 & 0.0 & 0.0 \\
\hline $\mathrm{VNNC}^{c}$ & 12 & 41.7 & 58.3 & 0.0 & 0.0 \\
\hline V-Test-ZS & 12 & 8.3 & 83.3 & 8.3 & 0.0 \\
\hline V+ZS-Test ${ }^{c}$ & 6 & 16.7 & 50.0 & 0.0 & 33.3 \\
\hline Controls & 10 & 100.0 & 0.0 & 0.0 & 0.0 \\
\hline
\end{tabular}

${ }^{a}$ VNNC and V-test-ZS groups are the same animals. ZS, Zinc sulfate infusion of the nasal cavities; VNNC and V, vomeronasal nerve cuts.

${ }^{b}$ Kill one pup, carry one pup.

${ }^{c} p<.01$ vs pooled control group. 
in latency to carry $(t(8)=4.26, p<.01)$ and increases in carrying efficiency $(t(8)=2.81, p<.05)$ were significant. The increase in duration carrying and pulling paper was not significant $(t(8)=.84, p>.2)$. There were no significant changes in killing behavior, although most animals decreased their latencies to attack and durations spent eating pups. Nest quality did not change uniformly in either group or both groups combined.

\section{Preoperative Carriers}

Olfactory bulbectomy. The postoperative responses to pups of all animals are shown in Table 1. Only one of the 14 bulbectomized (RBOB) pretest carriers continued to carry after bulbectomy $(p<.01$, Fisher's test vs pooled controls), and 4 now killed pups. Most of the animals neither killed nor carried either pup, although all of them sniffed the pups at least 19 times ( $M=34.6$ times for these nonresponders compared to 22.9 for controls). There were no differences in pretest behaviors between nonresponders and those that did exhibit a post-test response to the pups.

Only one of the 4 bulbectomy-induced killers killed both pups, although all but one attacked both. Because no untreated (Marques, 1976a) or sham-operated animals fail to kill pups which they attack, this result is due to the treatment. Killing was generally inefficient and poorly directed although both the latencies to attack $(M=14.3 \pm 4.9 \mathrm{sec})$ and average duration eating pups $(130 \mathrm{sec})$ were not different from pretest killers (both $p>.2)$.

Nest building was also inefficient and disorganized in these animals. Many of the nonresponders (6 of 9) did spend some time carrying or pulling paper $(M=19.9 \pm 9.1 \mathrm{sec})$. They did not, however, concentrate their nest building in one area of the cages for any length of time as did controls. As a result, none of these animals improved the quality of their nests during the test period as controls usually do.

Olfactory deafferentation. In general, zinc sulfate-treated animals did not appear sick when tested. They were not readily distinguishable from animals in other groups.

Eight of the 9 carriers with ZS treatment continued to carry in post-tests (Table 1). One animal killed one pup and carried the other. These carriers showed decreases in latencies to carry, increases in carrying efficiency, and increases in time spent carrying paper comparable to controls.

Vomeronasal deafferentation. All 10 females continued to carry after vomeronasal deafferentation (Table 1), and showed increases in maternal responsiveness similar to those seen in controls and ZS-treated animals. No comparisons with controls were significant.

Combined deafferentation. Combining ZS and VNNC did not produce any decreases in retrieval in preoperative carriers. Unlike after bulbectomy, no preoperative carrier became a nonresponder after combined deafferentation. One pretest carrier in the V+ZS-test group developed the 
intestinal disease "wet tail" (see Hoffman, Robinson, \& Magalhaes, 1968) shortly before testing and was not tested. All of the others carried both pups postoperatively, although one of these ate the second pup in the nest.

The rest of the pretest carriers showed near maximal maternal responsiveness after VNNC and ZS, with significant decreases in latency comparable to controls. There was an increase in paper pulling significantly greater than that seen in the pooled controls $(t(17)=2.23, p<.05)$.

$\mathrm{V}+\mathrm{ZS}$-Test animals were retested after the anosmic effect of the ZS treatment had worn off (see tests for olfaction). All showed the same response to pups as they had 2 days after ZS.

\section{Preoperative Killers}

Olfactory bulbectomy. Radical bulbectomies significantly reduced killing in animals which had been pretest killers $(p<.01$, Fisher's test vs controls). Only 6 of 11 of the pretest killers continued to kill, and 2 became carriers. These 2 had long latencies $(110,127 \mathrm{sec})$ and were inefficient (efficiency quotients $=0$ for both; mean of pretest carriers $=$ .47). Neither built a nest nor spent any time carrying or pulling paper. The other 3 bulbectomized animals neither killed nor carried pups.

Only 1 of the 6 killers failed to kill both pups. For these 6, there was no significant change in latencies to attack or in the number of bites.

Olfactory deafferentation. Five of the seven ZS-treated killers continued to kill in post-tests (Table 1). The other 2 now carried, but this change is not statistically greater than controls ( $p=.15$, Fisher's test). These two carriers were not different from the others in pretests.

In the 5 animals which killed in post-tests the latency to attack did not change but this did not differ significantly from controls. The decreased number of bites was similar to controls but the increase in time spent eating the pups was significantly greater than the increase by the pooled controls $(t(13)=2.44, p<.05$ for a comparison of increases).

Vomeronasal deafferentation. Interruption of the vomeronasal nerves converted $58 \%$ ( $p<.01$ vs controls, Fisher's test) of the killers into carriers (Table 1). The animals which became carriers were indistinguishable from the rest of the killers preoperatively. The 4 animals with the least damage to the main olfactory bulbs were all carriers in post-tests; and 3 of 5 with the most damage continued to kill. Therefore, the change to carrying was probably not a result of concomitant damage to the main olfactory bulbs.

The VNNC-induced carriers were as efficient as normal first-time carriers (mean latency $=49.6$ compared to 70.4 for normal first-time carriers-see Marques, 1976a-mean efficiency quotient $=.48$ compared to .47 for normals, both $p>.2$ ). The killers which were unchanged in their overt response to the pups were not different from controls. The latency 
of VNNCs to attack and the number of bites decreased sharply from pretest to post-test, comparable to controls.

Combined deafferentation. Adding ZS treatment to animals which have had VNNCs (V-test-ZS) further increases the number of killers which are converted to carriers (Table 1). The animals in the V-test-ZS group are the same as those in the VNNC group, now tested after additional zinc sulfate treatment. Only one of the killers now continued to kill. The 5 killer controls also given a third test all continued to kill $(p<.05$, Fisher's test). The other 4 animals which had killed after VNNC now carried pups with efficiency at least as good as normals (efficiency quotients $=.79$; mean latency $=20.5 \mathrm{sec}$ ). One animal, which had been converted to carrying after vomeronasal deafferentation, now neither killed nor carried. When the converted carriers were tested 1 week later (then able to smell) they continued to carry.

When animals are not allowed to have experience with pups between vomeronasal deafferentation and destruction of the olfactory mucosa $(\mathrm{V}+\mathrm{ZS}$-test), the results are not different from when they do have experience (Table 1), i.e., maternal responsiveness is increased. Only one of the 6 pretest killers in this group killed both pups: The other 5 now carried at least one pup ( $p=.001$, Fisher's test vs controls).

\section{Nest Building and Other Behaviors}

Radical bulbectomies produced significant decreases in nest quality (Table 2) and pretest killers and carriers did not differ in this effect. Almost $70 \%$ ( 16 of a possible 23; those 23 with pretest NQs of 1 or 2 ) of the animals built poorer nests than in pretests, and this decrease is significantly different from controls $\left(\chi^{2}=5.73, p<.05\right)$. One of a possible 16

TABLE 2

Change in Nest Quality from Pretests to Post-tests after Various Olfactory and Vomeronasal Disruptions ${ }^{a}$

\begin{tabular}{lcc}
\hline Treatment $^{b}$ & Percentage increasing $(n)$ & Percentage decreasing $(n)$ \\
\hline Bulbectomy & $6.3(16)$ & $69.6(23)^{c}$ \\
ZS & $30.0(10)$ & $0.0(11)$ \\
VNNC & $76.5(17)^{d}$ & $28.6(14)$ \\
V+ZS-Test & $69.2(13)^{e}$ & $10.0(10)$ \\
Controls & $27.2(11)$ & $35.7(14)$ \\
\hline
\end{tabular}

${ }^{a}$ Only those animals which could improve (i.e., pretest $N Q=0,1$ ) were included in the first column and only those which could show decreases (i.e., pretest $N Q=1,2$ ) were included in the second column. Some animals had pretest $N Q=1$ and appear in both columns.

${ }^{b}$ Both pretest killers and carriers are included in all groups.

${ }^{c} p<.01$ vs pooled control group.

${ }^{d} p<.03$ vs pooled control group.

${ }^{e} p<.05$ vs pooled control group. 
animals (those 16 with pretest NQs of 0 or 1 ) improved its nest quality after bulbectomy. Note that some animals had pretest NQs equal to 1 which could either increase or decrease and their results are therefore included in both columns.

Most ( 20 of $25,80 \%$ ) of the bulbectomized animals spent some time scrabbling $(M=50.3 \mathrm{sec})$, with all 20 showing an increase over pretests. The trend in controls was not as strong (43\% increased, $29 \%$ decreased; $p$ $<.05$ Fisher's test for bulbectomy vs controls). There was no change in the number of fecal pellets deposited.

ZS treatment had little effect on the quality of nest built. Only 3 of 10 received improved ratings in post-tests, and none of 11 ( $p=.08$, Fisher's test vs pooled controls) received decreased ratings (Table 2).

When all of the VNNC animals (both pretest killers and pretest carriers) are considered together for comparisons against the pooled control group, there is a significant increase in nest quality (Table 2) after VNNC. Most of the VNNC animals which could improve their nest quality (13 of 17) did so ( $p<.03$, Fisher's test vs controls), and only 3 of 14 built poorer nests after vomeronasal deafferentation. Very few of the carriers scrabbled and there was no change in scrabbling produced by VNNC on either the pretest killers or pretest carriers (means for both groups combined $=8.2$ sec pretest; 13.1 post-test).

$\mathrm{V}+\mathrm{ZS}$-Test animals also showed a statistically significant increase in the quality of nest built ( $p=.05$, Fisher's test, Table 2 ). There were no changes in scrabbling after combined deafferentation.

\section{Repeated Maternal Testing and Mating of Bulbectomized Animals}

Unlike controls, when radically bulbectomized animals were given a second postoperative test, they did not consistently show the same response to pups as in the first postoperative test. Six of the eleven radically bulbectomized animals tested in this way changed their responses to pups (Fig. 3), while none of the 5 controls did so. Two of the six bulbectomized animals changed back to the response they had shown in pretests.

After parturition, bulbectomized animals showed inefficient maternal behavior whereas controls (whether killers or carriers as virgins) immediately retrieved all of the pups to the nest. Only 4 of the 18 bulbectomized hamsters tested retrieved any pups to their nest within $5 \mathrm{~min}$ on at least 2 testing days, and only 5 animals had pups survive 5 days. The rest (13 of 18) killed and ate all of their pups within 1-2 days. The animals which showed good maternal behavior had all been killers on their postoperative tests as virgins.

Tests for olfaction. All of the controls tested in the food finding test located the food in less than $200 \mathrm{sec}$. In the food preference test all controls spent at least $8.3 \mathrm{sec}(M=31.0 \pm 4.3)$ sniffing at the hole above the food pellets. All but one of the controls spent over $60 \%$ of the total sniffing time (two holes combined) at the hole with the food under it. 

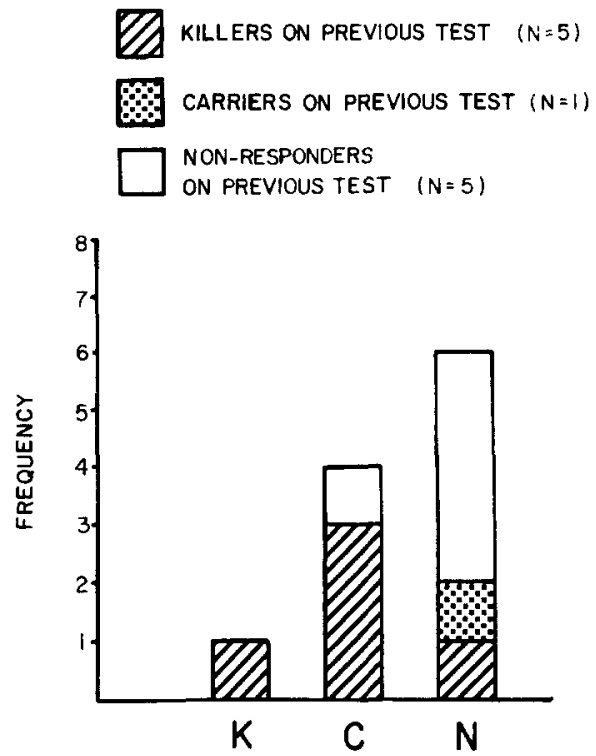

FIG. 3. Results of testing bulbectomized animals a second time postoperatively. Shading indicates the animals' responses on the first postoperative test. Most animals did not show the same overt response to pups on successive tests.

No radically bulbectomized animal spent more than $2.4 \mathrm{sec}$ sniffing at either hole. There is thus no overlap with controls.

Only two (both pre- and post-test carriers) of $16 \mathrm{ZS}$ animals were successful within the $300-\mathrm{sec}$ time limit in the food finding test. ZSTreated animals readily ate when given food pellets at the conclusion of testing.

Only two of the $14 \mathrm{~V}+\mathrm{ZS}$-test animals sniffed the food hole more than 8 sec (the minimum control value) and only one of them spent more time sniffing the food hole than the hole with wood under it. Both of these animals had been carriers in pretests and post-tests. The remaining $12 \mathrm{~V}+$ ZS-test animals spent an average of $2.5 \mathrm{sec}$ (range $=0-8.0$ ) sniffing the food hole. When tested 10 days later, just prior to the second postoperative test with pups, all $\mathrm{V}+\mathrm{ZS}$-test animals spent more than $9 \mathrm{sec}$ (range $=9.1$ to 45.5; $\mathrm{M}=22.9$ ) sniffing the food hole ( $p<.001$ sign test for test 1 vs test 2 ).

The V-test-ZS animal which had remained a killer throughout was the only V-test-ZS animal to sniff the food more than $5 \mathrm{sec}(13.5 \mathrm{sec})$. Except for that animal, there was again no overlap with controls.

\section{Discussion}

The reduction in pup killing produced by vomeronasal deafferentation and radical bulbectomy might represent a general interruption of coordinated attack or alternatively, a decrease in aggressiveness toward " prey" objects which might, for the virgin female hamster, include hamster pups. 
The inefficient disorganized behavior and lack of consistency from test to test after radical bulbectomy suggests a general disruption of organized behavior. The next experiment tests these interpretations.

\section{EXPERIMENT 2: OLFACTORY SYSTEMS AND CRICKET KILLING}

\section{Method}

Polsky (1976), Eibl-Eibesfeldt (1953), and Jacobs (1945) have observed that hamsters will readily attack, kill, and eat various insects including crickets. In this experiment, 66 animals from experiment 1 , some with each type of treatment (bulbectomy, ZS, VNNC, V+ZS-test, and controls), were presented with one cricket each, 1-2 days after post-tests with pups. Food had been returned to all animals following tests for olfaction. Animals were tested in the same procedure as they were with pups except that there was no nest paper, because crickets quickly hide.

Results

Only one of the 66 animals failed to catch and kill the crickets within 5 min, and the median latency from the first sniff to the kill was $2 \mathrm{sec}$. All 65 of the killers showed efficient, stereotyped attack directed toward the crickets, and all but 8 consumed most of the insect. Neither the olfactory nor the vomeronasal system is necessary for cricket killing in the female hamster.

\section{Discussion}

The approach and quick, predatory-like killing behavior pattern seen in spontaneous pup killers is still in the animals' behavior repertoire after complete removal of the olfactory systems. Most radically bulbectomized animals, however, did not attack and kill pups. This suggests that the nonresponders are not products of nonspecific reductions in the ability to perform coordinated, uninterrupted attacks. The first experiment demonstrated that the loss of sensory information was probably not causing the radically bulbectomized animals to be nonresponders. If there is no response deficit, and the behavior disruption is not caused by olfactory deficits, then the best conclusion is that radical bulbectomy has changed the perception or interpretation of nonolfactory stimuli coming from the pups, so that these stimuli no longer produce attack. Crickets may be more easily discriminable as prey (median latency $=2 \mathrm{sec}$ for cricket killing, $11 \mathrm{sec}$ for pup killing) and cricket killing may be dependent on fewer nonolfactory sensory cues than pup killing.

\section{GENERAL DISCUSSION}

Olfactory bulbectomy produced total or nearly total damage to both main and accessory olfactory bulbs. The olfactory peduncle was also 
damaged. All 25 bulbectomized animals appeared anosmic in tests with food odors. This type of damage has a complex effect on hamsters' responses to pups. One major effect is a nearly complete elimination of maternal behavior and nest building in experienced virgin females. This appears to be due to the effects of neural damage incurred by bulb removal rather than sensory loss, because it is not reproduced by either main olfactory receptor destruction (with zinc sulfate), or by vomeronasal deafferentation, or by the combined deafferentation.

Bulbectomy also reduces pup killing in experienced killers. About half (48\%) of the hamsters in this study with olfactory bulbs removed neither killed nor carried pups in a 10 -min testing period. This was not caused by a general response or motor deficit, as these animals efficiently killed crickets.

Another effect of olfactory bulbectomy is the induction of cannibalism in formerly maternal virgin hamsters. Although this result was not statistically significant in the present study, other reports (Marques, 1976a; Murphy, 1972) have shown a much stronger effect with somewhat less radical bulbectomies. In this study, increased cannibalism was not produced by sensory deficits in either the main olfactory or vomeronasal systems. This result parallels that of Fleming and Rosenblatt (1974b) who demonstrated that the increased cannibalism seen after bulbectomy was not found in zinc sulfate-treated animals.

The apparently contradictory finding that pup killing is induced in some pretest carriers and carrying is induced in some pretest killers can perhaps be resolved. Radically bulbectomized animals do not always show the same response to pups in repeated testing. It may be that bulbectomy results in the reduction of both carrying and killing, leaving neither response consistently dominant. This lack of a consistent response is also evident in the continuous interruption of several behavior patterns seen in bulbectomized animals in this test situation as well as in others (e.g., Goodman \& Firestone, 1973; Larue, 1975). These animals appear to continuously interrupt their own ongoing behavior with starts of other behaviors. A consequence of this self-interrupted pattern is that few behaviors (nest building, pup retrieval, pup killing) are completed, and none are efficiently accomplished.

Recent reports (e.g., Edwards, Schlosberg, McMaster, \& Harvey, 1977) have emphasized the difference between radical bulbectomies which damage much or all of the olfactory peduncle, and bulbectomies which are limited to more anterior portions of the olfactory bulbs and peduncle. The bulbectomies in the present paper are of the more radical type, and it might be argued that some of the nonsensory effects reported in this paper might not appear in animals with more conservative lesions. Preliminary results on seven hamsters with less extensive lesions suggest that this is not entirely the case (Marques, 1976a, 1976b). Pup carrying 
was eliminated in all preoperative carriers which had their olfactory bulbs removed but had most of the peduncle spared; six of the seven killed pups postoperatively. Nest building was also eliminated. This suggests that the elimination of pup retrieval and nest building seen after bulbectomy but not after peripheral sensory deafferentations is caused by destruction to the main olfactory bulbs. Damage to the posterior parts of the olfactory system (the olfactory peduncle, primarily) may, however, be responsible for reductions in pup killing in spontaneous killers and for the extreme behavioral disorganization seen in most radically bulbectomized animals.

Peripheral sensory deafferentation of the vomeronasal system has a dramatic effect which is very different from that of bulb removal. After vomeronasal nerve cuts, half of the animals which had previously killed pups became carriers. Pup retrieval was as efficient as in spontaneously maternal animals. This effect was probably not due to incidental main olfactory bulb damage. Generally, those hamsters which changed their behavior had less main bulb damage than those which continued to kill.

Vomeronasal deafferentation also produced a change in nest quality ratings (Table 2). These ratings are assigned before the pups are introduced and might be thought to provide evidence for a nonsensory role of the vomeronasal organ. However, nonpup chemical stimuli in the environment may influence the maternal responsiveness of hamsters through the vomeronasal organ. The vomeronasal system might monitor chemical stimuli in the environment which inform the animal of the appropriateness of that environment for maternal behavior, nest building, or cannibalistic behavior.

Neither pup carrying nor pup killing was greatly affected by olfactory deafferentation produced by intranasal ZS treatment. ZS-treated carriers still carried, most ZS-treated killers still killed, and none became nonresponders. The ZS treatment was identical to that used by Powers and Winans (1973) who showed that male hamsters' ability to discriminate between amyl acetate and female hamster vaginal secretion was completely lost 2 days after ZS. Hamsters recovered, however, within 5-10 days. Olfactory localization and preference tests done on animals in this experiment yielded essentially the same conclusions.

It has been demonstrated that some ZS treatments do not always produce complete olfactory deficits in rats (Slotnick \& Gutman, 1977). Although there is strong evidence that the procedure used here does produce anosmia for up to 5 days in hamsters (Powers \& Winans, 1973), it must be considered possible that some olfactory capabilities remained in the hamsters tested in this study.

This conclusion does not alter the interpretation of the results. Although the ZS-induced sensory deficit by itself produced minimal changes in maternal responsiveness, ZS treatment did appear to produce an in- 
crease in maternal responsiveness when done in combination with vomeronasal deafferentation. This was reflected by the increased conversion of killers to carriers and by the very large increase in time spent nest building in the V-test-ZS group. Because these animals showed normalappearing coordinated behaviors, a general debilitation (caused by sickness from zinc sulfate) explanation seems ruled out. That this effect is caused by partial, if not complete, sensory loss is the best interpretation.

This interaction of main olfactory and vomeronasal deafferentations is analogous to the results of Powers and Winans (1975) and Winans and Powers (1977) which demonstrated that ZS treatment had no effect on male hamster mating behavior when administered alone, yet produced a deficit when combined with vomeronasal deafferentation. One difference is that the change in behavior produced by the additional ZS treatment in this experiment had a permanent effect outlasting the apparent sensory loss.

Combined peripheral deafferentation $(\mathrm{VNNC}+\mathrm{ZS})$ does not account for the major effects of bulbectomy. There were no decreases in retrieval efficiency, no changes in scrabbling, no evidence of disorganized behavior, and only one nonresponder. Nest quality ratings actually improved after peripheral deafferentations and no animals showed the selfinterrupted behavior patterns so characteristic of bulbectomized animals. These differences may be the results of differences between the sensory and nonsensory (Cain, 1974) effects of bulbectomy.

Note added in proof. Since the original presentation of this research in 1976 (Marques, 1976b) others have shown similar effects of vomeronasal system disruption on maternal behavior in the rat. While this paper was under review, Fleming, Vaccarino, Tambosso, and Chee (1979) published a paper demonstrating that vomeronasal deafferentation reduces the latency of virgin female rats to show retrieval of pups and a nursing posture. They suggest that the effects of vomeronasal nerve destruction and main bulb damage are additive in the facilitation of maternal responsiveness in the rat. No effects on cannibalism and nest building were reported.

\section{REFERENCES}

Benuck, I., \& Rowe, F. A. (1975). Centrally and peripherally induced anosmia: Influences on maternal behavior in lactating female rats. Physiology and Behavior, 14, 439-447.

Cain, D. P. (1974). The role of the olfactory bulb in limbic mechanisms. Psychological Bulletin, 81, 654-671.

Davis, B. J., Macrides, F., Youngs, W. M., Schneider, S. P., \& Rosene, D. L. (1978). Efferents and centrifugal afferents of the main and accessory olfactory bulbs in the hamster. Brain Research Bulletin, 3, 59-72.

Edwards, D. A., Schlosberg, A. J., McMaster, S. E., \& Harvey, J. A. (1977). Olfactory system damage and brain catecholamines in the rat. Brain Research, 121, 121-130.

Eibl-Eibesfeldt, I. (1953). Zur Ethologie des Hamsters (Cricetus cricetus L.). Zeitschrift für Tierpsychologie, 10, 204-254. 
Fleming, A. S., \& Rosenblatt, J. S. (1974). Olfactory regulation of maternal behavior in rats. I. Effects of olfactory bulb removal in experienced and inexperienced lactating and cycling females. Journal of Comparative and Physiological Psychology, 86, 221-232. (a)

Fleming, A. S., \& Rosenblatt, J. S. (1974). Olfactory regulation of maternal behavior in rats. II. Effects of peripherally induced anosmia and lesions of the lateral olfactory tract in pup induced virgins. Journal of Comparative and Physiological Psychology, 86, 233246. (b)

Fleming, A., Vaccarino, F., Tambosso, L., \& Chee, P. (1979). Vomeronasal and olfactory system modulation of maternal behavior in the rat. Science, 203, 372-374.

Gandelman, R., Zarrow, M. X., Denenberg, V. H., \& Myers, M. (1971). Olfactory bulb removal eliminates maternal behavior in the mouse. Science, 171, 210-211.

Goodman, E. D., \& Firestone, M. I. (1973). Olfactory bulb lesions, nest reinforcement, and handling reactivity in hamsters. Physiology and Behavior, 10, 1-8.

Hoffman, R. A., Robinson, P. F., \& Magalhaes, H. (Eds.). (1968). The Golden Hamster: Its Biology and Use in Medical Research. Ames: Iowa State Univ. Press.

Jacobs, D. L. (1945). Food habits of the golden hamster. Journal of Mammalogy, 26, 199.

Johns, M. A., Feder, H. H., Komisaruk, B. R., \& Meyer, A. D. (1978). Urine-induced reflex ovulation in anovulatory rats may be a vomeronasal effect. Nature (London), 272, 446-447.

Larue, C. (1975). Prandial drinking and the disruption of meal patterns in olfactory bulbectomized rats. Physiology and Behavior, 15, 491-493.

Leonard, C. (1972). Effects of neonatal (day 10) olfactory bulb lesions on social behavior of female golden hamsters (Mesocricetus auratus). Journal of Comparative and Physiological Psychology, 80, 208-215.

Marques, D. M. (1976). Determinants of maternal behavior and cannibalism in the female golden hamster (Doctoral dissertation, University of Michigan, 1976). Dissertation Abstracts International, 1977, 5423B-5424B, (University Microfilms No. 77-7984). (a)

Marques, D. M. (1976). Separate olfactory and neural systems mediate maternal behavior, cannibalism, and cricket killing in the female hamster. Neuroscience Abstracts, 2, 158. (b).

Marques, D. M., \& Valenstein, E. S. (1976). Another hamster paradox: More males carry pups and fewer kill and cannibalize young than do females. Journal of Comparative and Physiological Psychology, 90, 653-657.

Murphy, M. R. (1972). Different Effects of Olfactory Bulb Removal on Different Kinds of Aggression in Male Syrian Golden Hamsters. Unpublished doctoral dissertation, Massachusetts Institute of Technology.

Polsky, R. H. (1976). Conspecific defeat, isolation/grouping, and predatory behavior in golden hamsters. Psychological Reports, 38, 571-577.

Powers, J. B., \& Winans, S. S. (1973). Sexual behavior in peripherally anosmic male hamsters. Physiology and Behavior, 10, 361-368.

Powers, J. B., \& Winans, S. S. (1975). Vomeronasal organ: Critical role in mediating sexual behavior of the male hamster. Science, 187, 961-963.

Raisman, G. (1972). An experimental study of the projection of the amygdala to the accessory olfactory bulb and its relationship to the concept of a dual olfactory system. Experimental Brain Research, 14, 395-408.

Rowell, T. E. (1961). Maternal behaviour in non-maternal golden hamsters. Animal Behavior, 9, 11-15.

Scalia, F., \& Winans, S. S. (1976). New perspectives on the morphology of the olfactory system: Olfactory and vomeronasal pathways in mammals. In R. L. Doty (Ed.), Mammalian Olfaction, Reproductive Processes, and Behavior, pp. 7-28. New York: Academic Press. 
Seegal, R. F., \& Denenberg, V. H. (1974). Maternal experience prevents pup killing in mice induced by peripheral anosmia. Physiology and Behavior, 13, 339-341.

Slotnick, B. M., \& Gutman, L. A. (1977). Evaluation of intranasal zinc sulfate treatment on olfactory discrimination in rats. Journal of Comparative and Physiological Psychology, 91, 942-950.

Vandenbergh, J. G. (1973). Effects of central and peripheral anosmia on reproduction of female mice. Physiology and Behavior 10, 257-261.

Winans, S. S., \& Powers, J. B. (1977). Olfactory and vomeronasal deafferentation of male hamsters: Histological and behavioral analysis. Brain Research, 126, 325-344. 